\title{
eJRIEPS
}

Ejournal de la recherche sur l'intervention en éducation physique et sport

19 | 2010

Varia

\section{Panorama des biennales de l'ARIS de 2000 à 2008}

\section{Mathilde Musard, Marie-Paule Poggi et Nathalie Wallian}

\section{CpenEdition \\ Journals}

Édition électronique

URL : http://journals.openedition.org/ejrieps/5457

DOI : 10.4000/ejrieps.5457

ISSN : 2105-0821

Éditeur

ELLIADD

\section{Référence électronique}

Mathilde Musard, Marie-Paule Poggi et Nathalie Wallian, « Panorama des biennales de l'ARIS de 2000

à 2008 », eJRIEPS [En ligne], 19 | 2010, mis en ligne le 01 janvier 2010, consulté le 08 janvier 2021

URL : http://journals.openedition.org/ejrieps/5457 ; DOI : https://doi.org/10.4000/ejrieps.5457

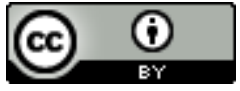

La revue eJRIEPS est mise à disposition selon les termes de la Creative Commons Attribution 4.0 International License. 
Mathilde Musard, Marie-Paule Poggi \& Nathalie Wallian

GRIAPS/LaSeIDi (EA 22-81)

UFR STAPS de Besançon, Franche-Comté

\section{Résumé}

Cette étude s'inscrit dans la première mission de l'ORIS (Observatoire des Recherches sur l'Intervention en Sport) «Mutualiser, caractériser et synthétiser les connaissances produites " et vise à construire un état des lieux des communications produites au cours des cinq premiers congrès ARIS de 2000 à 2008. L'article se centrera sur les questions suivantes : qui participe aux colloques ARIS ? Quelles sont les finalités des recherches? Quels champs scientifiques et quelles méthodologies sont mobilisés pour étudier l'intervention ? L'analyse de contenu quantitative des programmes scientifiques ( $n=730$ résumés) a été réalisée à l'aide du logiciel Sphinx (6 dimensions d'analyse ; 22 items). Les résultats questionnent les relations entre pratique, formation et recherche, dans la mesure où les communications présentées sont essentiellement des recherches, de nature descriptive et compréhensive. D’autre part, les chercheurs s'appuient sur différents champs scientifiques et méthodologies. Cette diversité est synonyme de richesse dans le champ de l'intervention, car le croisement de différentes approches théoriques et méthodologiques, toutes complémentaires les unes des autres, permet d'accéder à une connaissance de plus en plus fine de l'intervention en sport.

\section{Introduction}

Depuis 1999, une communauté de professionnels et chercheurs francophones intéressés par l'intervention en sport s'est constituée autour de l'Association pour la recherche sur l'intervention en sport (ARIS), suite à l'organisation de plusieurs congrès autour des questions de l'intervention (Toulouse en 1996: “Actualités de la recherche en EPS; Louvain-la-Neuve (1998) : “Identifier les effets de l'intervention en motricité humaine »; Antibes en 1998 : "Recherche sur l'intervention en éducation physique et en sport»). La notion d'intervention s'impose progressivement comme une notion clé dans le domaine des sciences de la motricité, du sport et de la formation. «Elle désigne toute action et situation individuelle ou collective à destination d'une ou plusieurs personnes engagées dans une activité physique sportive et artistique (APSA), et visant des modifications de 
cette activité. Ces actions sont de natures différentes en fonction des contextes au sein desquels elles se déroulent, des personnes auxquelles elles s'adressent, des intentions des intervenants et de la nature des modifications envisagées. Ces intentions peuvent être d'éducation, de rééducation, d'entraînement et de performance, d'entretien et de loisir, de récupération, etc... » (Durand, 1998).

Cette association s'intéresse aux diverses facettes de l'intervention et organise tous les deux ans une biennale dans le monde francophone. Les missions de l'ARIS sont déclinées sur le site internet de l'association :

- «promouvoir et faire connaître les actions de recherche portant sur le système de l'intervention dans le domaine des activités physiques et sportives ainsi que de la motricité humaine (enseignement de l'éducation physique, entraînement sportif, animation des loisirs, réadaptation par les activités physiques et sportives) ;

- faciliter et développer les relations entre tous les chercheurs et les praticiens intéressés à ces recherches dans le domaine des activités physiques et sportives, en suscitant des échanges et en soulignant la richesse des acquis professionnels ;

- assurer une aide au niveau de la définition de programmes de recherche finalisés par l'intervention, de la diffusion et de l'exploitation de leurs résultats ;

- établir de bonnes relations avec d'autres associations de chercheurs d'activités physiques et sportives, dans un souci de valorisation des recherches sur l'Intervention sportive. 》

Force est de constater que les travaux produits dans le champ de l'intervention en sport sont de plus en plus nombreux : au sein de l'ARIS, 730 papiers ont été sélectionnés par les comités scientifiques des cinq congrès. Ainsi, il devient urgent de construire un panorama visible des divers travaux réalisés au sein de l'ARIS. Catégoriser les études en croisant différentes dimensions d'analyse, mettre en réseau, mutualiser et synthétiser les connaissances, les méthodes et les résultats permettra à chacun de se positionner par rapport aux diverses tendances qui se profilent dans le champ de l'intervention.

C'est ainsi que l'ARIS a décidé de créer en 2007 l'Observatoire des Recherches sur l'Intervention en Sport (ORIS). En France, il existe de multiples observatoires, souvent créés par les collectivités et l'État. Ces observatoires ont pour mission de structurer les connaissances et de les communiquer sous une forme synthétique afin de faciliter les prises de décisions et de faciliter l'accès à l'information dans différents domaines (social, économique, environnement..). II en existe à toutes les échelles territoriales, les plus pérennes et nombreux étant nationaux, régionaux ou départementaux. Dans le monde de l'éducation, de nombreux observatoires ont été créés pour décliner avec rigueur et 
précision les évolutions d'innovations et de recherches (l'observatoire européen de la recherche universitaire, l'observatoire des sciences et techniques, l'observatoire des technologies pour l'éducation en Europe, l'observatoire des réformes en éducation, l'observatoire des pratiques enseignantes, l'observatoire européen de la violence en milieu scolaire, l'observatoire des politiques d'éducation, l'observatoire européen des innovations en éducation et en formation...). Ceux-ci permettent de mieux connaître certains objets en rassemblant et en diffusant des savoirs sur un sujet donné à destination d'un large public. Un observatoire peut être défini comme un dispositif mis en œuvre par plusieurs partenaires pour suivre l'évolution d'un ou plusieurs phénomènes dans l'espace et dans le temps. L'ORIS est donc un dispositif d'observation qui a pour vocation d'analyser, de décrire et d'interpréter l'évolution des Recherches et Innovations sur l'Intervention en Sport en contextes variés (enseignement de l'éducation physique et sportive, entraînement sportif, animation des loisirs, réadaptation par les activités physiques et sportives...) et de les projeter dans l'avenir. Véritable outil de veille scientifique, il permet de caractériser les activités de recherche et d'innovation en émergence, en transformation ou en obsolescence et de nourrir ainsi en permanence les formations professionnelles. Cet outil d'information et d'aide à la décision se situe d'emblée dans la durée, avec l'ambition de déboucher sur le long terme sur une réelle et fructueuse interaction entre recherche et formation.

L'ORIS s'est attribué plusieurs missions :

1.Mutualiser, caractériser et synthétiser les connaissances produites ;

2.Développer des réflexions de nature épistémologique ;

3.Faire reconnaître les travaux sur l'intervention et les projeter dans l'avenir, développer des collaborations avec différents partenaires.

Les objectifs relatifs à chacune de ces trois missions sont ici déclinés :

\section{Mutualiser, caractériser et synthétiser les connaissances produites}

II s'agit de proposer un état des lieux des travaux existants à partir de plusieurs filtres d'analyse (objets d'étude, visée des recherches, cadres théoriques, méthodologies employées, résultats...). Inventorier, caractériser les productions de l'ARIS, rassembler les connaissances scientifiques éparpillées permettra d'établir une typologie des recherches et d'offrir une vision plus synthétique et lisible du champ de l'intervention (a). II semble essentiel de cumuler les données d'enquête sur une période longue pour effectuer des comparaisons dans le temps et ainsi repérer des évolutions (b). Enfin, positionner les études menées dans le monde francophone par rapport à la littérature anglophone 
favorise l'ouverture vers d'autres cadres théoriques et facilite les échanges et les débats dans les congrès internationaux (c).

\section{Développer des réflexions de nature épistémologique}

Les travaux issus de cette première mission, à savoir les états des lieux à propos des recherches sur l'intervention en sport, devraient nourrir des questionnements épistémologiques: comment se construisent les connaissances scientifiques? Plus précisément, à quels champs scientifiques et à quels cadres théoriques les chercheurs et professionnels se réfèrent-il pour étudier l'intervention (a) ? Quelles sont les principales méthodologies de recherche employées (b) ? Quelles sont les relations entre chercheurs et professionnels au cours du processus de recherche ? (c) Quels critères de scientificité élaborer pour les recherches en intervention qui analysent des pratiques autonomes, incertaines et complexes (d) ?

3.Faire reconnaître les travaux portant sur l'intervention, les partager avec différents partenaires et les projeter dans l'avenir.

Cette troisième mission de l'ORIS est déterminante pour que la recherche soit ancrée dans les différents milieux sociaux. Un premier objectif consiste à travailler pour que les recherches sur l'intervention soient mieux reconnues par les institutions (scientifiques, politiques...) (a). II devient urgent d'affirmer et de défendre d'autres critères de scientificité que ceux du courant positiviste pour les recherches qui étudient des pratiques humaines. Le second objectif vise à multiplier les échanges entre professionnels (enseignants, entraîneurs, éducateurs,...), formateurs, chercheurs et étudiants pour que la recherche vive et ne se coupe pas des pratiques (b). Comment les recherches peuvent-elle nourrir les différentes formations (formation initiale, formation continue, formation de formateurs) ? Comment expliquer les difficultés liées à l'interaction formation recherche et quels dispositifs de collaboration professionnels - chercheurs encourager pour que formation et recherche s'enrichissent mutuellement? Comment organiser davantage de rencontres, initier plus de discussions entre les divers acteurs pour fournir une aide à la décision aussi bien pour les intervenants, les formateurs et les chercheurs ?

Cet article s'inscrit dans la première mission de l'ORIS (1. (a) « Mutualiser, caractériser et synthétiser les connaissances produites »). Des chercheurs ont déjà proposé un bilan des orientations de la recherche en EPS (Marsenach \& Amade-Escot, 1993 ; David, Bouthier, Marsenach \& Durey, 1999 ; Bouthier, 2000 ; Amade-Escot \& Amans-Passaga, 2006). II s'agit ici de proposer un état des lieux des cinq premiers congrès ARIS en s'intéressant aux différents champs d'intervention. L'analyse quantitative cherche à répondre aux 
questions suivantes: qui participe aux colloques ARIS ? Quelles sont les finalités des recherches ? Quels champs scientifiques sont mobilisés dans l'analyse des pratiques d'intervention ? A l'aide de quelles méthodologies ? Un second article dans ce numéro se focalise sur la description des objets d'étude investigués dans les recherches sur l'intervention en sport.

A travers cette étude, nous montrerons que si les recherches sur l'intervention francophones s'intéressent à des objets variés en s'appuyant sur une pluralité de cadres théoriques et méthodologiques, elles semblent néanmoins se caractériser par certaines tendances. En comparant les données de cet observatoire avec les travaux américains (Kulinna \& al., 2009 ; Ward \& Ko, 2006 ; Silverman \& Skonie, 1997 ; Silverman \& Manson, 2003 ; Macdonald \& al., 2002), nous mettrons en évidence certaines spécificités des recherches francophones. En effet, celles-ci s'appuient sur des perspectives théoriques et méthodologiques particulières; elles s'orientent essentiellement vers des finalités compréhensives et des méthodes qualitatives. Face à ces spécificités, nous pouvons nous demander si les recherches sur l'intervention en sport peuvent être considérées comme des constructions sociales (Goodson, 1988). Certes, la dimension sociale est constitutive de l'activité scientifique. Berthelot (2008) s'arme d'ailleurs d'un triple point de vue pour spécifier l'activité scientifique comme activité sociale, en combinant les apports de la philosophie et de la sociologie :

(1) l'activité scientifique est soumise à des normes collectives, qu'elles soient intellectuelles, institutionnelles ou culturelles. Ainsi, pour être reconnu comme enseignantchercheur en intervention, il convient de présenter ses travaux selon certaines étapes et certaines règles de recevabilité et de scientificité, de respecter des modèles légitimes à un moment donné ;

(2) l'activité scientifique est nécessairement intégrée à une situation d'interaction, directe ou indirecte, qui engendre des règles régissant les comportements. Les interactions entre les acteurs, depuis les échanges informels au sein d'un laboratoire jusqu'aux débats lors des colloques nationaux ou internationaux, participent du développement de l'activité scientifique ;

(3) l'activité scientifique est orientée vers autrui, avec une intentionnalité. Les recherches sont soumises à autrui pour être expertisées, puis elles sont diffusées à travers les congrès, les formations et les revues scientifiques ou professionnelles.

Si ces trois positions (institutionnaliste / interactionniste / intentionnaliste) renvoient à des cadres théoriques différents, nous pouvons toutefois admettre que « des agents intentionnels tissent entre eux, dans les situations où ils se trouvent, des interactions avec 
leurs règles propres, tout en s'insérant dans des institutions et des organisations définissant un cadre normatif global » (p. 11).

Pouvons-nous pour autant affirmer que la science est une «construction sociale » ? Si l'expression de « construction sociale » a connu dans les années 1980 et 1990 un essor considérable dans des domaines très variés depuis la parution de l'ouvrage fondateur $L a$ construction sociale de la réalité de Berger \& Luckmann en 1966, elle est désormais controversée. La notion de construction sociale est devenue une idée vague, imprécise et creuse, qui oscille entre banalité («tout est construit, rien n'est objectif») et radicalité (« rien n'est faux, rien n'est vrai»). Ainsi, le philosophe et historien des sciences Hacking fait remarquer dans son ouvrage The social construction of what? (2001) que cette expression n'est pas toujours utilisée de façon pertinente, selon que l'on s'intéresse aux objets au sens large (les personnes, les objets matériels, les pratiques, les classes) ou bien aux idées que l'on se fait de ces objets (concepts, théories, croyances). II propose alors un espace où peuvent coexister et s'articuler réel et construit social. Dans la même lignée, Lahire (2005) met en évidence dans un chapitre de son ouvrage L'esprit sociologique les «splendeurs et misères d'une métaphore : « la construction sociale de la réalité » ». Les constructions sociales seraient des créations intersubjectives fragiles et éphémères, " où tout se rejouerait à chaque instant, où tout se réinventerait à chaque interaction avec des acteurs et dans des contextes singuliers ॥ (p. 104). II n'est pourtant pas possible de nier le poids des dispositions incorporées et des dispositifs objectivés. En effet, si les pratiques de recherche se construisent en fonction de variables contextuelles (par exemple les débats lors de congrès), elles dépendent également étroitement de l'histoire du champ scientifique considéré et de celle des acteurs (connaissances, expériences...). Peut-on considérer que la science ne serait en réalité qu'une construction sociale comme une autre, qu'un discours sur le réel, reflétant des croyances partagées et intéressées par un groupe donné à un moment donné ? Les constructions scientifiques reposent sur davantage de réflexivité, d'explicitation et de preuves argumentatives et empiriques que les autres constructions, moins exigeantes du point de vue de l'effort de la démonstration (Lahire, 2005). De même, Berthelot (2008) défend un modèle d' « emprise du vrai », alternatif au logicisme et au constructivisme social : l'activité scientifique n'est pas une activité sociale comme les autres, elle présente une certaine spécificité en cherchant à produire des connaissances de plus en plus rationnelles. Finalement, elle relève d'une pragmatique, (c'est-à dire d'une théorie d'action) qui se veut simultanément sociale, historique, contextualiste mais aussi rationaliste. Si les enseignants-chercheurs reconstruisent, recomposent progressivement des programmes de recherches plus ou 
moins novateurs à partir de différentes théories ou de nouvelles connaissances scientifiques, c'est bien dans le but de produire des connaissances de plus en plus rationnelles sur l'intervention.

\section{Méthodologie de la recherche}

Le corpus a été constitué à partir des programmes scientifiques des cinq biennales de I'ARIS :

1.Colloque de Grenoble "L'intervention dans le domaine: compétence(s) en mutation ? », 14-15-16 Décembre 2000 : 144 résumés ;

2.Colloque de Rennes «Cultures Sportives et Artistiques - Formalisation des Savoirs professionnels - Pratiques, Formations, Recherches », 12 - 13 - 14 Décembre 2002 : 139 résumés ;

3. Colloque de Louvain - La - Neuve «Intervenir dans les activités physiques, sportives et artistiques - Pratiques - Recherches - Formations », 20-21-22 Janvier 2005 : 138 résumés

4.Colloque de Besançon «Co-construire des savoirs: les métiers de l'intervention dans les APSA », 9-10-11-12 Mai $2006: 157$ résumés ;

5. Colloque de Rodez «L'intervention en sport et ses contextes institutionnels : cultures et singularité de l'action », 14 - 15 - 16 Mai 2008 : 149 résumés.

L'analyse de contenu (Bardin, 2001 ; Weill-Barais, 1997) des 730 résumés a été réalisée à l'aide du logiciel Sphinx. Après plusieurs lectures flottantes, 22 items sous forme de questions fermées (essentiellement à choix multiples) ont été renseignés à travers cinq dimensions d'analyse :

1.les informations permettant de situer la communication (congrès, nom, sexe de l'auteur principal, pays, institutions) ;

2.la nature de la communication (recherche, compte-rendu d'innovation, revue de littérature, point de vue personnel, réflexion épistémologique, présentation d'équipe de recherche) ;

3.la visée dominante de la recherche (évaluer l'efficacité des pratiques, transformer les pratiques, décrire et/ou expliquer pour comprendre les pratiques) ;

4.la méthodologie de la recherche (les techniques de recueil des données, le croisement de différentes méthodes) ;

5.le thème de la recherche, en fonction du contexte d'intervention analysé (EPS, formation, entraînement...). 
L'analyse quantitative des données a consisté en des tris à plat (calcul des fréquences) et tris croisés (test du chi2) pour repérer d'éventuelles relations significatives entre variables (influence des variables « congrès », «pays », « sexe », « laboratoire »...). D'autre part, nous avons souhaité investiguer une sixième dimension d'analyse, celles des cadres théoriques mobilisés par les communiquants. Compte tenu de la multiplicité des théories et concepts, cités de façon plus ou moins explicite, nous n'avons pu traiter cet item sous forme de question à choix multiples. C'est pourquoi nous nous sommes tournés vers l'analyse lexicale pour identifier les auteurs les plus cités dans les résumés. Si les occurrences d'auteurs ne sont pas satisfaisantes pour décrire la diversité des références théoriques, elles permettent dans un premier temps de repérer les principaux champs scientifiques mobilisés pour étudier les pratiques d'intervention.

\section{Résultats}

Dans cette partie, nous répondrons aux questions suivantes : (1) qui participe à l'ARIS ? (2) Quelle est la nature des communications présentées ? (3) Quelles sont les finalités des recherches présentées lors des biennales de l'ARIS ? (4) Quels champs scientifiques sont mobilisés pour analyser les pratiques d'intervention ? (5) Quelles méthodologies de recherche sont plus particulièrement utilisées pour analyser l'intervention ?

3. 1. L'ARIS, une communauté internationale en essor

Lors de chaque biennale, l'ARIS rassemble plus de 200 francophones autour d'un nombre conséquent de communications (entre 130 et 160, réparties en conférences, communications orales, communications affichées, ateliers, symposia...). Une communauté solide s'est donc progressivement constituée pour réfléchir et échanger sur des problématiques liées à l'intervention en sport. Ce réseau stabilisé de chercheurs et de professionnels révèle l'importance des enjeux scientifiques, sociaux et de formation des recherches sur l'intervention.

Parmi les communiquants aux cinq congrès de l'ARIS, plus d'un quart provient d'autres pays que la France, notamment du Canada (11,1\%) et de la Belgique (9,5\%) (figure 1). Au total, ce sont 18 nationalités qui sont représentées. 


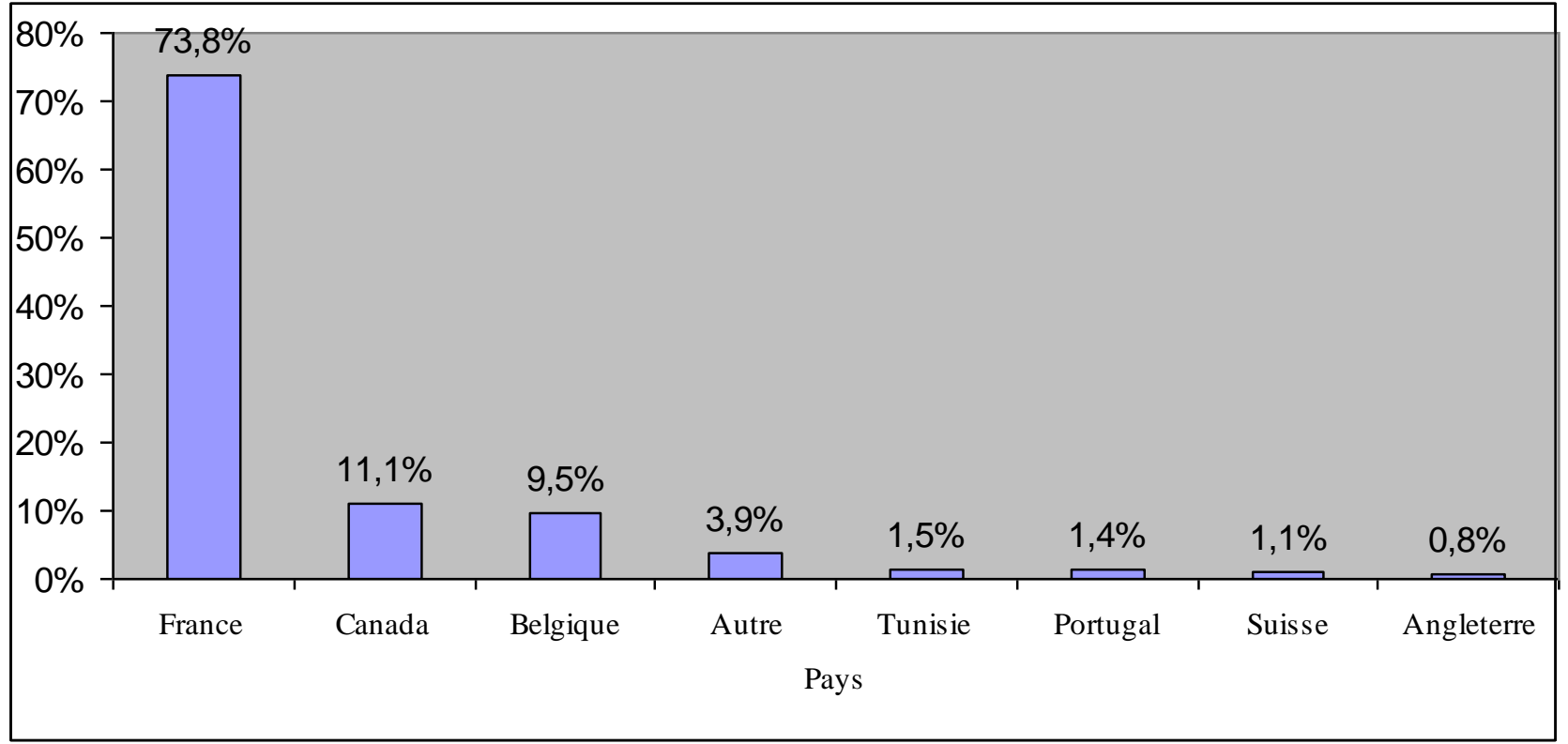

Figure 1. Nationalités des communiquants aux cinq biennales de l'ARIS.

Catégorie "Autre »: Angleterre, Taiwan, USA, Espagne, Roumanie, Bulgarie, Irlande, Algérie, Brésil, Chili, Allemagne, Sénégal.

Force est de constater que les congrès ARIS sont de plus en plus internationaux : la diminution sensible et régulière du pourcentage de français (de $81 \%$ en 2000 à $63,8 \%$ en 2008) révèle une participation de plus en plus importante des autres pays $(15,4 \%$ de canadiens et $13,4 \%$ de belges au dernier colloque de Rodez en 2008). Ces colloques permettent aux chercheurs d'approfondir la littérature étrangère, d'échanger sur des travaux portant sur les mêmes thématiques, mais mobilisant des cadres théoriques et des méthodologies variés, mais aussi de comparer des résultats de recherches, dépendants de spécificités culturelles. Cette diversité est d'ailleurs une source d'enrichissement mutuel; pour preuve, la collaboration internationale entre différents laboratoires de recherche s'étend et produit un certain nombre de publications à l'ARIS.

Notons que plus d'une centaine de laboratoires de recherche participe aux congrès. Une quinzaine d'équipes de recherche (universités/IUFM de Besançon, Bordeaux, ClermontFerrand, Grenoble, Louvain-La-Neuve, Laval, Liège, Lille, Lyon, Orléans, Ottawa, Nantes, Paris 12, Rennes, Sherbrooke, Toulouse...) s'est spécialisée dans le domaine de l'intervention et a publié au moins une vingtaine de communications au cours des cinq congrès ARIS. Les équipes de recherche francophones, spécialistes de l'intervention se développent donc peu à peu, mais restent très localisées, encore loin d'être représentées dans toutes les universités. Kirk, Macdonald \& O'Sullivan (2006) montrent dans l'introduction de The Handbook of physical education que si le champ de la recherche en éducation s'est bien développé comme en témoigne l'existence de différentes associations 
internationales de recherche, les chercheurs doivent continuer de se battre dans les universités du monde entier pour défendre ce champ encore marginalisé.

Qui participe aux congrès ARIS (figure 2) ?

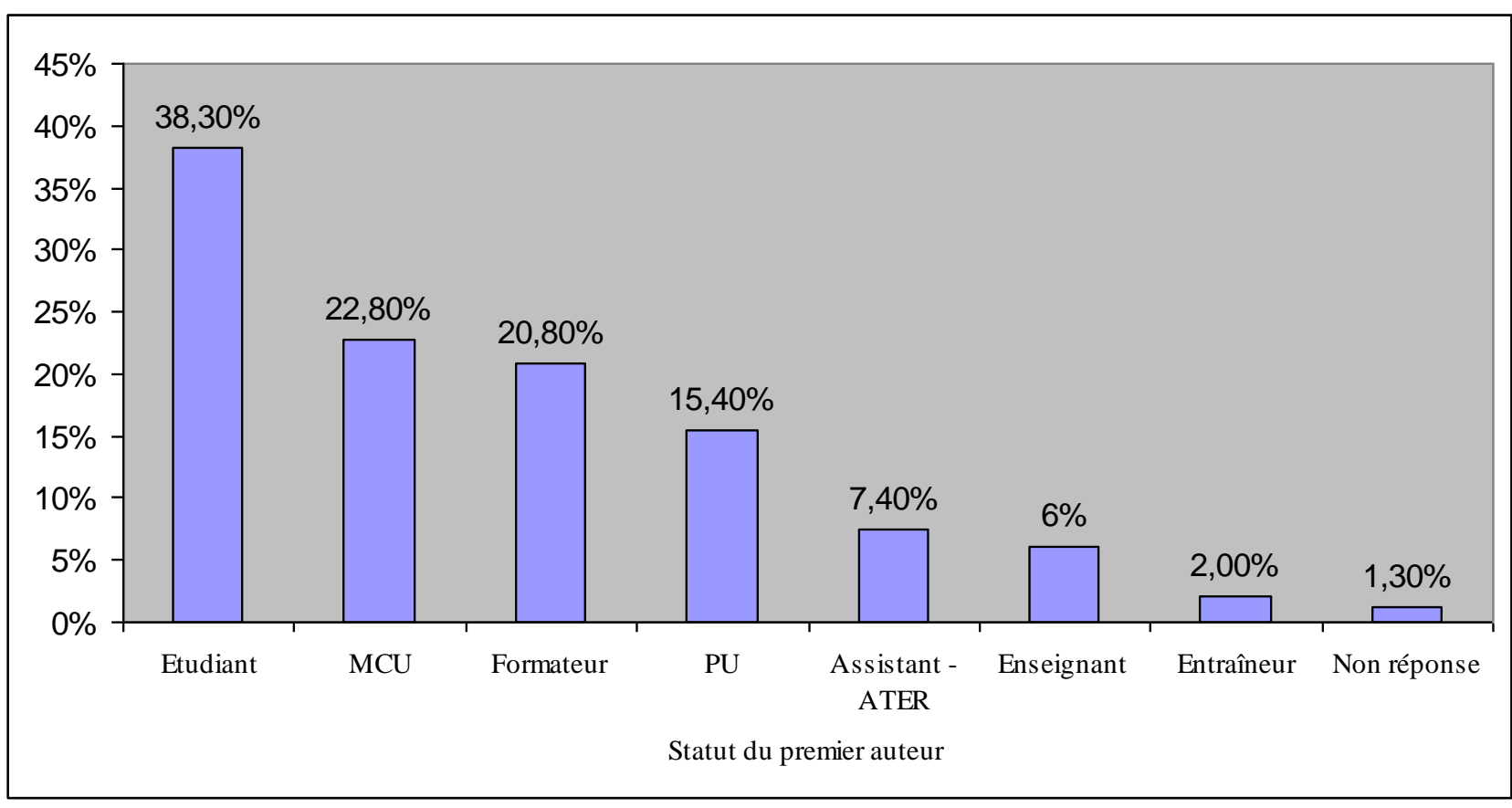

Figure 2. Statut du premier auteur communiquant lors du congrès ARIS de Rodez (2008).

Remarque : un communiquant peut avoir simultanément deux fonctions, par exemple formateur et doctorant, assistant et doctorant...

Lors du dernier congrès de Rodez (2008), nous constatons que les étudiants (en thèse pour la plupart, voire en master pour une minorité) et les assistants ou attachés temporaires d'enseignement et de recherche (ATER) représentent $45,7 \%$ des premiers auteurs communiquants. Ils sont donc plus nombreux que les enseignants-chercheurs (38,2\%, en réunissant maîtres de conférences des universités (MCU) et professeurs des universités (PU)). Ces données confirment que les recherches en intervention sont en plein essor dans le monde francophone, comme dans le monde anglophone. En effet, le nombre de revues de recherche en intervention, de publications et d'étudiants de $3^{\mathrm{e}}$ cycle ne cesse d'augmenter (Kulinna \& al., 2009 ; Macdonald \& al., 2002).

Enfin, 20,8\% des premiers auteurs sont des formateurs, enseignant le plus souvent à l'université. La plupart des formateurs s'est d'ailleurs engagée dans des activités de recherche, voire a soutenu un doctorat; d'autres présentent des comptes-rendus d'expériences. Là aussi, cette donnée est encourageante car les formateurs constituent un maillon incontournable dans l'articulation entre recherche et formation. En revanche, nous reviendrons plus tard dans cet article sur l'inquiétante sous-représentation des 
professionnels de l'intervention, puisque seulement 6\% sont des publiants sont enseignants dans le premier ou second degré et $2 \%$ sont entraîneurs.

D'autre part, un tiers seulement des communiquants est féminin. C'est surtout lors du premier colloque à Grenoble que les femmes étaient sous-représentées (22,2\%), puisqu'elles étaient plus de $33 \%$ lors des quatre congrès suivants (voir figure 3 ). Ward et Ko (2006) observent également les pourcentages d'hommes et de femmes publiant en premier auteur dans la revue américaine JTPE (Journal of Teaching in Physical Education) et relèvent une répartition équilibrée entre hommes et femmes à partir des années 90. De 1980 à 1990, les auteurs masculins étaient bien plus nombreux, car les femmes ont intégré les formations académiques bien plus tard que les hommes. Nous pouvons alors nous interroger sur les raisons de cette sous-représentation des femmes publiantes dans le monde francophone, comme d'ailleurs dans de nombreuses disciplines.

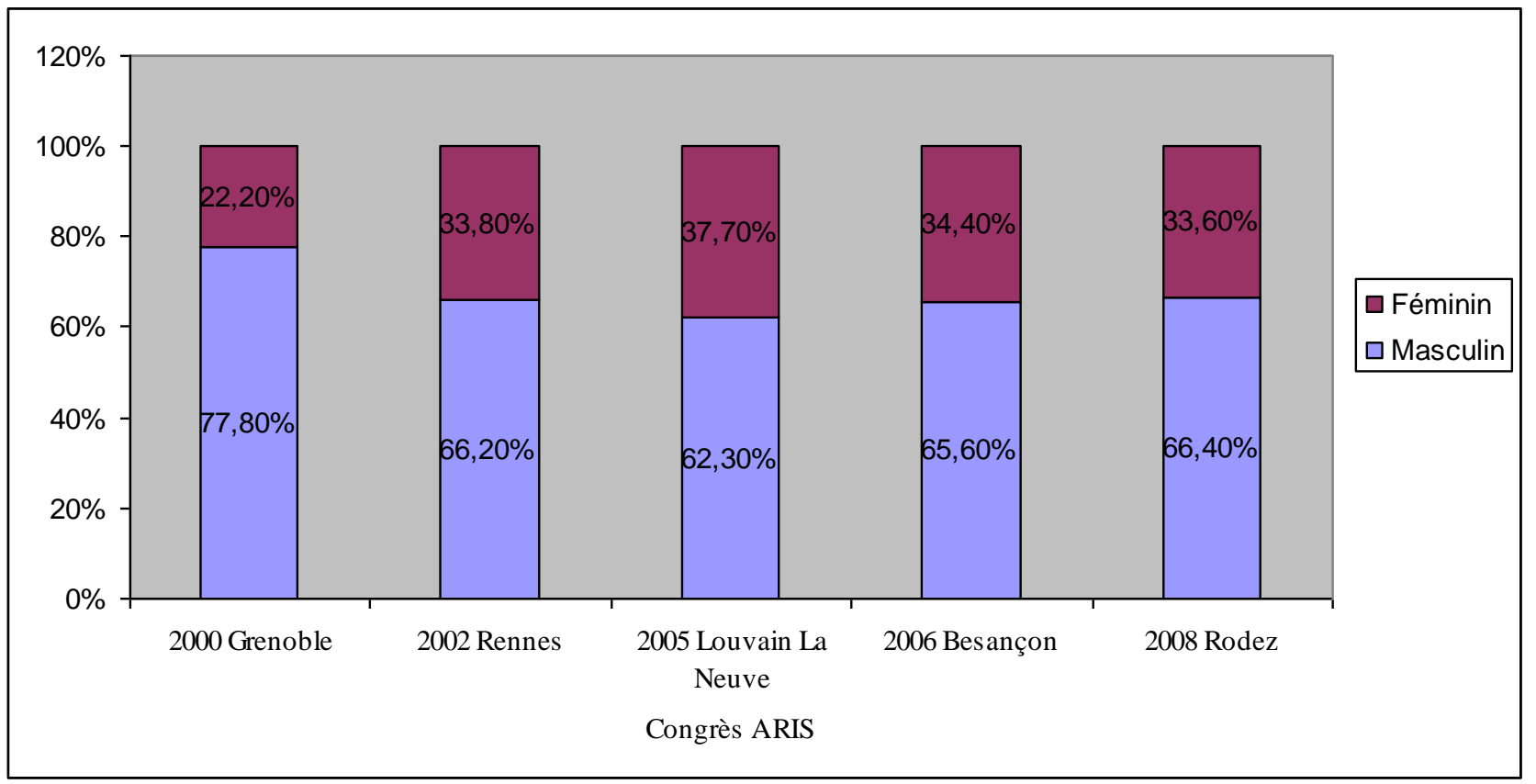

Figure 3. Sexe du premier auteur selon les congrès ARIS.

3. 2. Evolution de la nature des communications selon les congrès

La figure 4 montre qu'un nombre croissant de participants choisit d'exposer lors des congrès ARIS les différentes étapes de sa recherche (cadre théorique, problématique et hypothèses - méthodologie de recueil et de traitement des données - résultats discussion - conclusion). En effet, les doctorants, les enseignants-chercheurs et les formateurs qui enseignent à l'université et réalisent en parallèle un travail de thèse sont de 
plus en plus nombreux, ce qui explique la place croissante des recherches (de $55,6 \%$ en 2000 à $88,6 \%$ en 2008).

En revanche, les réflexions de nature épistémologique, qui portent par exemple sur la pertinence de certaines approches théoriques ou méthodologiques, sur le statut ou la subjectivité du chercheur, sont en baisse significative (de 19,4\% à $2 \%$ ), alors que ces questions se posent sans cesse dans le métier de chercheur.

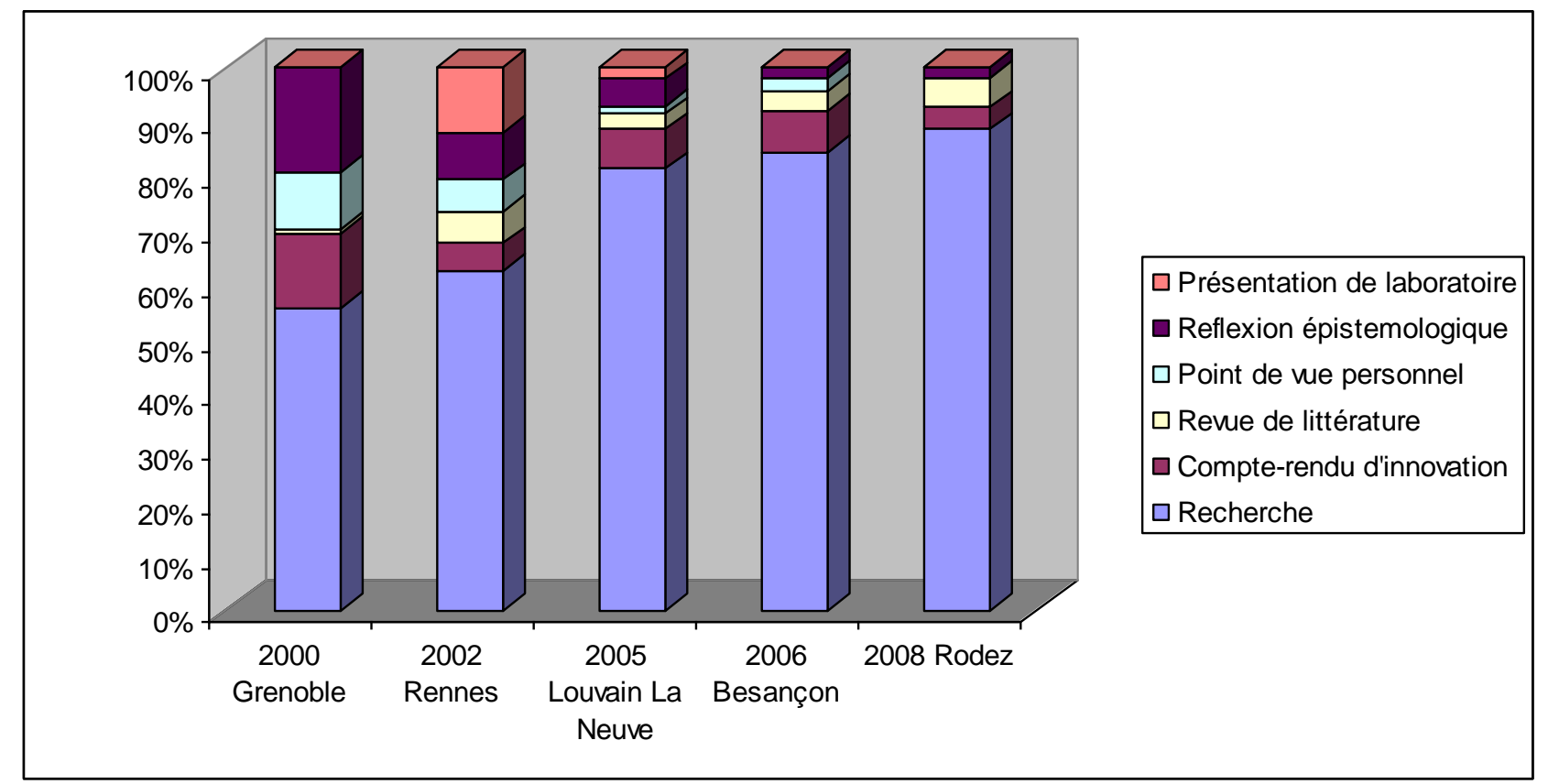

Figure 4. Nature des communications selon les congrès.

Si l'on peut se satisfaire aujourd'hui de l'augmentation conséquente des études scientifiques dans le champ de l'intervention en sport, on peut néanmoins s'interroger quant à la diminution significative des comptes-rendus d'innovation (description d'un dispositif innovant, du contexte d'intervention et analyse des effets produits) et des points de vue personnels (expression d'un point de vue à propos des métiers d'intervenants, des cultures physiques et sportives, des formations...), respectivement de $13,9 \%$ à $4 \%$ et de $10,4 \%$ à $0 \%$. Certains professionnels, en particulier les formateurs et les professeurs d'EPS, se sont tournés vers la recherche pour analyser de façon plus objective un problème particulier et présentent désormais leurs résultats de recherche, plutôt qu'un compte-rendu d'innovation ou leur point de vue personnel. Mais comment expliquer que professionnels de l'intervention et chercheurs ne se rencontrent pas davantage pour échanger sur des problématiques relatives à l'intervention en sport ? Les modalités de présentation des recherches (destinées le plus souvent à un public déjà familiarisé à la recherche) et d'organisation des congrès (peu d'espaces plus spécifiquement adressés 
aux praticiens: ateliers technologiques, tables rondes réunissant chercheurs et professionnels...), les représentations de la recherche, comme « coupée » des pratiques, les finalités essentiellement descriptives et compréhensives des recherches, le faible nombre de recherches collaboratives entre praticiens et chercheurs sont des facteurs qui peuvent expliquer la baisse de fréquentation des professionnels.

3. 3. Les finalités des recherches

En nous inspirant des catégorisations de Bru (2002) et Astolfi (1993), nous avons distingué trois finalités des recherches: (1) évaluer l'efficacité des pratiques, (2) transformer les pratiques et innover, (3) décrire/expliquer pour comprendre les pratiques. Chacune de ces trois finalités est ici déclinée à partir de recherches présentées lors des congrès ARIS :

\section{Evaluer l'efficacité des pratiques:}

Plus précisément, ces recherches ont pour but de :

- comparer différentes modalités d'intervention et identifier les plus performantes, évaluer les effets des pratiques des intervenants sur les apprentissages (pratiques constructivistes vs pratiques technicistes en sports collectifs, impact du débat d'idées et de la verbalisation sur les apprentissages, étude des conditions les plus favorables au travail par tutorat, effets des formes de groupement sur les progrès des élèves, effets de différents modèles de démonstration sur les apprentissages d'habiletés gymniques, utilisation de l'athlétisme pour développer la vitesse des footballeurs...);

- mesurer les effets d'un programme d'activité physique et sportive (développement d'habiletés physiques, de compétences), les impacts d'enseignements visant l'éducation à la santé ou de programmes d'entraînement (effets de l'entraînement à la prise de décision en volley-ball sur les performances des joueurs), ou encore les effets de la pratique sportive sur le niveau scolaire ;

- tester l'impact de formations innovantes sur le développement de l'activité professionnelle (dispositif de conseil pédagogique, stage de formation professionnelle continue, dispositif interdisciplinaire de formation initiale, formation au soutien de l'autonomie, cyber-enseignement) ;

- tester des sujets (performance, compétences, savoirs...);

- évaluer une structure (les besoins des entraîneurs et des athlètes, la nécessité d'emplois sportifs dans les institutions spécialisées, l'impact d'un sport-études sur la vie d'un établissement) ; 
- évaluer la fiabilité d'outils (par exemple le TSAP: Team Sport Assessment Procedure) pour mesurer les performances de joueurs en contexte réel.

2. Transformer les pratiques, innover ; expliciter les données d'un problème et contribuer à sa résolution (recherches à visée praxéologique) :

Ces innovations concernent :

- les traitements didactiques des APSA (par exemple la dialectique technique artistique dans les activités gymniques ou artistiques, le pentathlon en équipe pour inciter les jeunes à la pratique d'activité physique), les compétences et les contenus à développer (la $5^{\mathrm{e}}$ compétence culturelle des programmes de lycée (CC5), la santé, l'autonomie, l'estime de soi, la construction de contenus significatifs pour les élèves en course de haies) et la mise en œuvre de dispositifs (par exemple les formats de jeu évolutifs adaptés aux différents profils de compétiteurs en tennis) ;

- la formation des intervenants et des formateurs (les dispositifs de recherches collaboratives pour améliorer l'intervention ou la formation, la rénovation de la situation traditionnelle de conseil pédagogique, l'observation didactique au service du développement de compétences professionnelles de formateurs, la place des savoirs technologiques dans la formation, l'aide aux enseignants débutants pour dépasser leurs dilemmes) ;

- la mise en œuvre de nouveaux outils pour aider les intervenants (analyse du jeu, modélisation des conduites typiques, grilles de lecture des techniques, conceptions de situations d'apprentissage à partir de l'interprétation des comportements des élèves, outils informatiques, classification d'éléments techniques ou d'habiletés, coconstruction d'un référentiel commun en rugby à l'aide de verbalisations, évaluation...).

Les recherches-action (Van Der Maren, 1995), les recherches d'ingénierie didactique (Artigue, 1990) et les recherches technologiques (Bouthier \& Durey, 1994) s'inscrivent dans cette visée de transformation des pratiques.

\section{Décrire et/ou expliquer pour comprendre les pratiques :}

Ces recherches à visée heuristique se centrent sur :

- l'activité des intervenants (planification, gestion des groupes, instruction, régulation, modalités de communication, évaluation, conceptions, savoirs professionnels, développement professionnel) ; 
- l'activité des pratiquants (expériences, perceptions, stratégies, interactions langagières...) ;

- les APSA (programmes, finalités, compétences, contenus, méthodes, évaluation) ;

- les interactions entre intervenant - pratiquant - APSA.

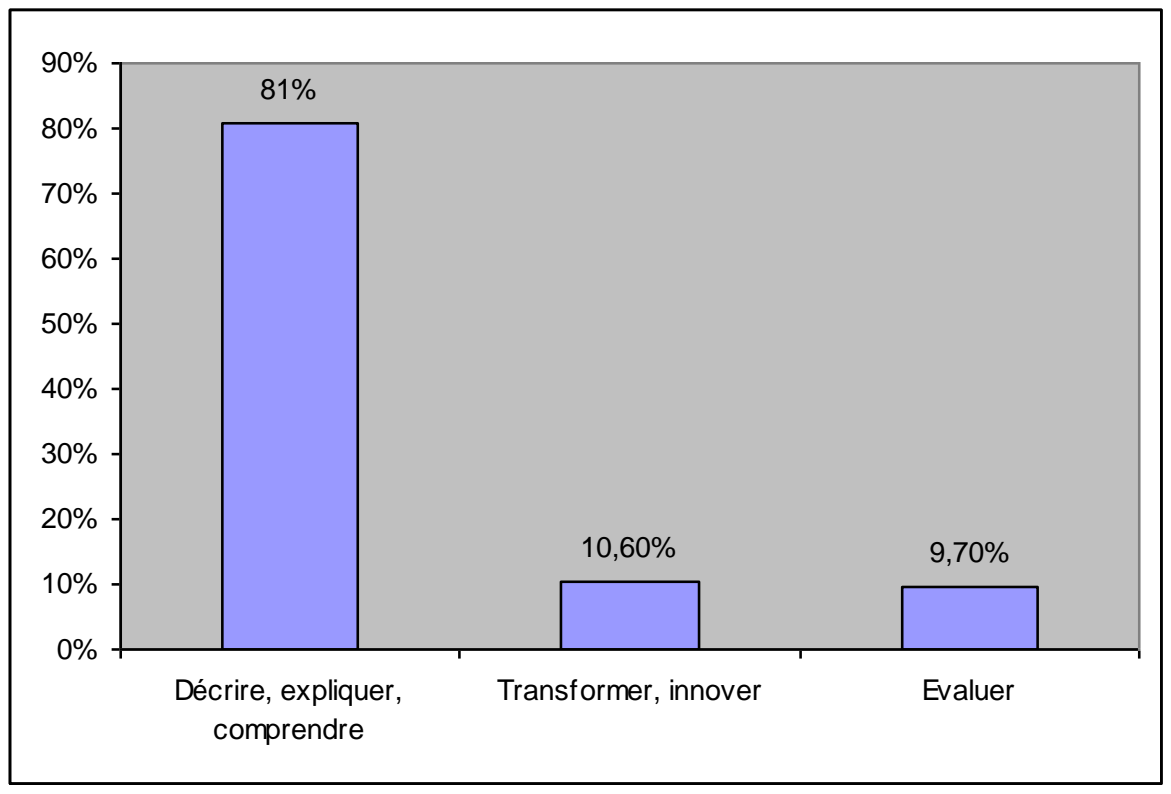

Figure 5. Visée dominante des recherches présentées lors des cinq biennales

Plus de $80 \%$ des recherches ARIS sont à visée heuristique. Ces données sont stables et n'évoluent pas de façon significative en fonction des congrès ou des domaines étudiés (EPS, entraînement, formation...). Face à des pratiques complexes, incertaines et autonomes, les chercheurs ne s'autorisent pas à prescrire des solutions ou des "recettes» aux praticiens, car la généralisation de résultats obtenus dans un contexte singulier reste très problématique. C'est pourquoi ils s'orientent de plus en plus vers des recherches descriptives/explicatives et compréhensives, qualitatives, ancrées dans des contextes authentiques. Ils souhaitent saisir la complexité des pratiques humaines et décrire très finement, par des études de cas, l'activité des individus en contexte naturel. Ces recherches s'avèrent indispensables, car l'action se construit en partie en fonction de variables contextuelles. Les recherches expérimentales, dans lesquelles un certain nombre de variables est contrôlé dans le but d'obtenir des résultats généralisables, sont donc plus rares.

Cette orientation des recherches francophones vers les recherches descriptives et compréhensives contraste nettement avec les recherches américaines. En effet, Silverman \& Manson (2003) analysent plus de 500 thèses en EPS de 1985 à 1999 et 
soulignent la suprématie de recherches centrées sur l'efficacité de l'enseignant (92,5\%), dont $42,8 \%$ de recherches centrées sur la comparaison de différentes méthodes d'enseignement et $23,4 \%$ de recherches descriptives, alors que $4 \%$ des recherches portent sur la cognition de l'enseignant et 3,5\% sur le développement d'outils pour l'intervention. A noter qu'une autre étude de Silverman \& Skonie (1997) portant sur 179 articles de recherche en EPS publiés entre 1980 et 1994 conclut à des tendances similaires. Selon nous, la différence dans les périodes étudiées ne peut expliquer à elle seule cette importante variation des finalités des recherches : les recherches descriptives représentent $83 \%$ des publications ARIS en EPS, alors qu'elles comptent pour moins d'un quart des recherches aux EU. A l'inverse, les chercheurs américains sont beaucoup plus centrés sur l'efficacité de l'enseignement que les francophones (8\% pour les recherches francophones en EPS). Nous pouvons faire l'hypothèse que les façons de concevoir les recherches sont situées historiquement et culturellement. Chaque champ de recherche a sa propre trajectoire (Kirk, Macdonald \& O'Sullivan, 2006), développe certains paradigmes en fonction du contexte politique, culturel, historique et social du pays.

Enfin, le nombre important de recherches heuristiques dans le monde francophone peut nous questionner, puisque celles-ci interrogent sous un angle particulier l'articulation recherche et formation. Nous pouvons nous demander si davantage d'équilibre entre les trois types de recherches évoqués ne serait pas souhaitable, dans la mesure où ceux-ci sont complémentaires dans le champ de l'intervention. En effet, les recherches descriptives et compréhensives sont sans doute moins utilisées par les professionnels, car elles débouchent rarement sur des propositions très concrètes d'intervention. La relation entre recherche et formation semble peut-être plus directe dans le cas des recherches à visée praxéologique. Pourtant, les recherches heuristiques pourraient constituer des ressources pertinentes pour questionner les pratiques d'intervention et donc à terme les transformer. Si les formateurs peuvent jouer un rôle charnière décisif entre intervenants et chercheurs en s'appuyant sur les recherches pour analyser les pratiques, les chercheurs peuvent aussi s'investir dans cette articulation recherche - formation en participant à des formations de formateurs, en développant des recherches collaboratives ou encore en présentant leurs travaux de recherche de façon plus didactique aux professionnels.

3. 4. Les champs théoriques mobilisés

Quels sont les auteurs les plus cités dans les résumés des communications ? A quels champs scientifiques appartiennent-ils? 


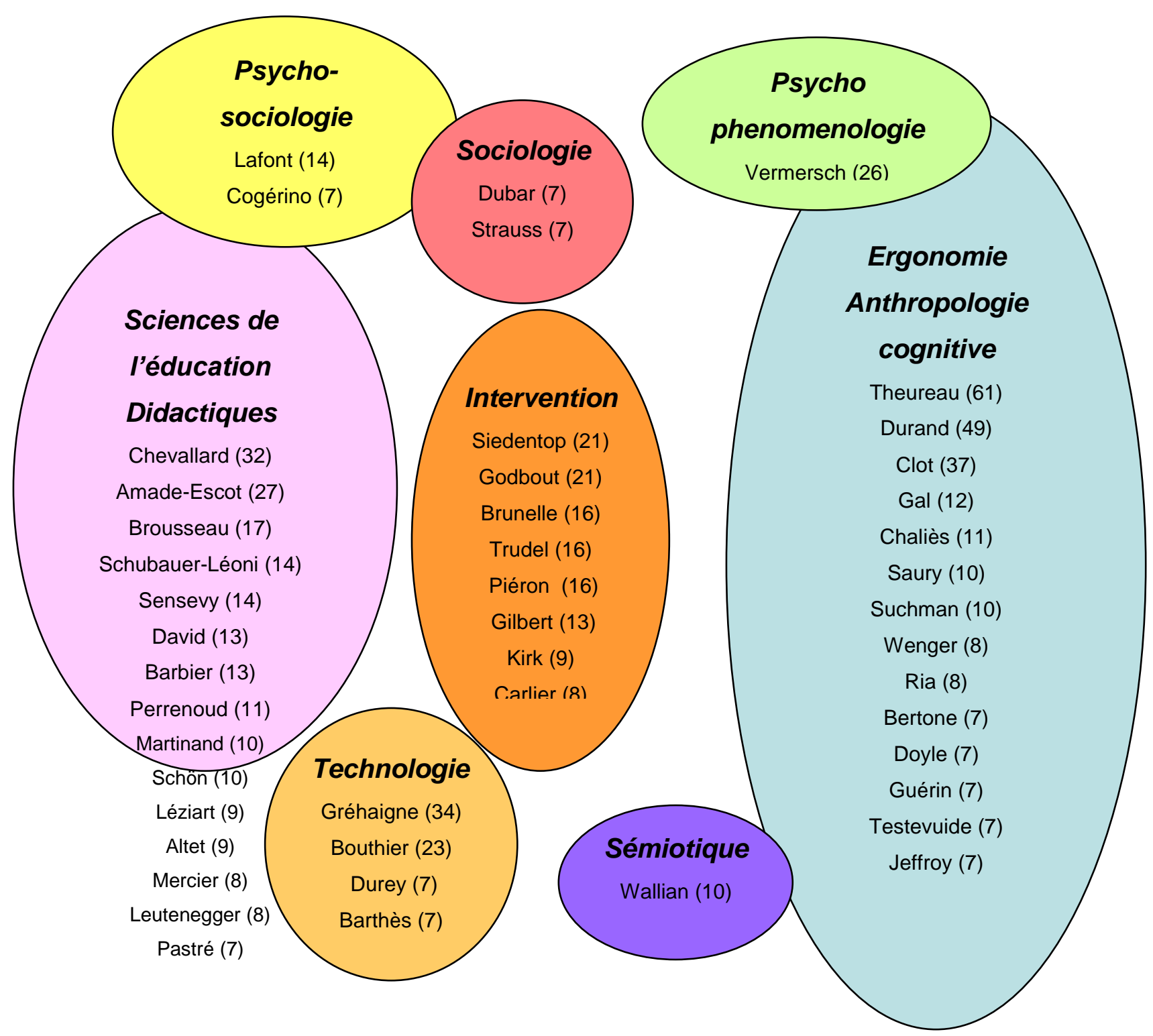

Figure 6. Auteurs cités dans les résumés des congrès ARIS (à partir de 7 occurrences)

Rappelons que cette figure a été élaborée à partir des auteurs les plus fréquemment cités (à partir de 7 occurrences) dans les résumés des programmes scientifiques. Ce travail exploratoire est donc loin d'être suffisant pour présenter les champs théoriques mobilisés dans les recherches en intervention (une analyse qualitative à partir d'articles plus longs mériterait d'être développée), mais il permet néanmoins de donner quelques tendances. En effet, nous constatons que divers champs théoriques sont utilisés par les chercheurs francophones pour analyser les mêmes objets d'étude, allant des sciences de l'éducation et des didactiques à la cognition située, en passant par la psychologie, la sociologie ou la sémiotique... En fonction des laboratoires de recherche auxquels ils appartiennent, de leurs convictions théoriques, les chercheurs développent donc des programmes de 
recherche sur l'intervention diversifiés et complémentaires. D'ailleurs, Macdonald et al. (2002) soulignent également la variété des paradigmes développés dans les recherches anglophones, différents des paradigmes francophones (positiviste, interprétatif, social, poststructuraliste, féministe). Cette diversité dans les façons de penser, d'analyser l'intervention, de récolter et de traiter les données est synonyme de richesse, car elle apporte une complémentarité et une connaissance plus approfondie des phénomènes d'intervention.

3. 5. Vers un croisement des méthodologies de recherche

La figure 7 illustre les méthodes de recueil de données utilisées dans les recherches en intervention. Deux techniques sont largement répandues avec une différence significative : l'entretien $(57,8 \%)$ et l'observation $(55,6 \%)$. Les questionnaires, les traces écrites (documents, programmes, articles...) et les tests sont beaucoup moins utilisés (respectivement $22,1 \%, 14,4 \%$ et $6,8 \%$ ). Cette tendance est stable de 2000 à 2008, puisqu'on n'observe pas de différence significative selon les congrès.

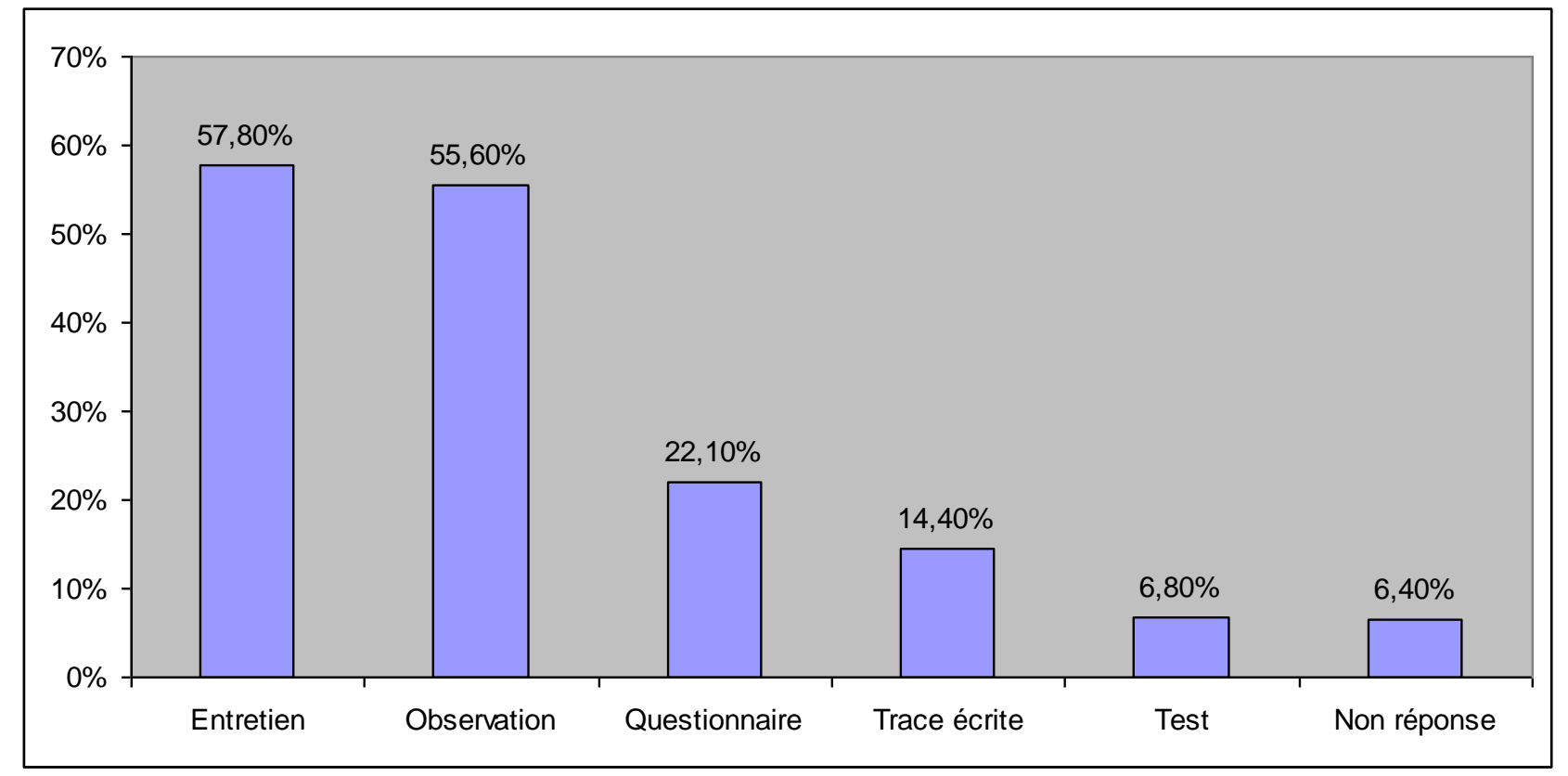

Figure 7. Méthodes de recueil de données

Ainsi, les chercheurs tentent d'approcher au plus près la complexité des pratiques, conscients que l'observation comportementale ne suffit plus pour comprendre l'activité des acteurs. D'ailleurs, des recherches croisent au moins deux techniques différentes. Dans $39 \%$ des recherches, c'est un couplage observation - entretien qui est choisi. Le plus souvent, il s'agit de l'observation en situation naturelle croisée avec l'entretien d'autoconfrontation ou l'entretien semi-directif. D'autres formes de triangulation sont moins 
souvent utilisées, par exemple les couplages entretien - analyse de documents (14,6\%), entretien - questionnaire (13,9\%) ou observation - analyse de documents (12,2\%).

Cette «triangulation » de données extraites de sources différentes constitue, comme le soulignent Huberman \& Miles (1991) et Van Der Maren (1995) une stratégie pertinente de validation en recherche qualitative. « La triangulation consiste à retracer la chaîne la plus causale possible (...) en s'efforçant d'obtenir plus d'un type de mesure à partir de plus d'une source pour chaque maillon de la chaîne » (Huberman \& Miles, 1991, p. 427). Elle est supposée confirmer un résultat en montrant que les mesures indépendantes qu'on en a fait vont dans le même sens, ou tout au moins ne se contredisent pas et permet de mieux apprécier la crédibilité des résultats.

Quelles formes d'entretien et d'observation les chercheurs choisissent-ils pour analyser les pratiques d'intervention?

Si l'on se focalise sur la strate des recherches (316 communications) qui ont mobilisé l'entretien (annexe 1), on observe une variété de techniques (plus d'une quinzaine de types d'entrevues). Deux techniques, l'entretien d'auto-confrontation (32,6\%) et l'entretien semi-directif $(25,3 \%)$ sont utilisées de manière significative. Les chercheurs s'appuient dans une moindre mesure sur des entretiens ante ou post leçon / cycle (10,8\%) et sur les entretiens d'explicitation (8,2\%). Les autres techniques (récits de vie, incidents critiques, rappel stimulé...) sont plus rares (moins de $5 \%$ ).

L'entretien semi-directif ou semi-dirigé représente une technique flexible très utilisée, qui se caractérise par le degré de liberté accordé à l'enquêté. Ainsi, l'entretien est ni complètement directif, structuré, ni complètement libre. Le chercheur dispose d'un certain nombre de thèmes ou de questions guides, relativement ouvertes, qui ne sont pas posées dans un ordre strict et sous leur formulation exacte, mais plutôt en fonction du flux conversationnel et des réactions de l'interlocuteur. Autant que possible, le chercheur laisse venir l'interviewé afin que celui-ci puisse parler ouvertement, dans les mots qu'il souhaite et dans l'ordre qui lui convient. II essaie simplement de recentrer l'entretien sur les thèmes qui l'intéresse quand l'entretien s'en écarte, et de poser les questions auxquelles l'interviewé ne vient pas par lui-même. Les données recueillies au cours d'entretiens semi-directifs peuvent être très diverses: opinions, croyances, représentations, connaissances, ....

Les entretiens ante ou post leçon / cycle sont utilisés dans les études de contrats didactiques. L'entretien ante cycle vise à questionner l'enseignant sur ses objectifs de cycle, la structure de son cycle, ses conceptions à propos de l'activité enseignée. 
L'entretien ante leçon est centré sur les caractéristiques des tâches d'apprentissage (contenus, variables, matériel, durée..). Les entretiens post leçon ou post cycle cherchent à recueillir à chaud le bilan, l'analyse réflexive du professeur à propos de sa leçon ou de son cycle (stratégies des élèves, progrès..).

L'entretien semi-directif ne vise pas forcément le retour de l'acteur sur un moment particulier de son action/activité, à la différence de l'entretien d'auto-confrontation (Theureau, 1992) et de l'entretien d'explicitation (Vermersch, 1994). Rix (2006) souligne les convergences et différences de ces deux formes particulières d'entretien. D'une part, elles présentent plusieurs fondements épistémologiques communs :

- l'acteur est considéré comme un praticien réflexif, capable de re-connaître -au sens de connaître à nouveau sous un autre jour, dans une autre posture- sa propre activité ;

- le retour de l'acteur sur sa propre activité soulève certaines difficultés comme la prise de conscience de son expérience subjective; les méthodologies d'autoconfrontation et d'explicitation visent alors toutes deux à aider au retour et à la verbalisation de l'action visés.

D'autre part, dans le cas de l'entretien d'auto-confrontation, on demande à l'acteur de commenter l'enregistrement vidéo de son comportement immédiatement après son action pour saisir ce qui est pré-réflexif, significatif, montrable, racontable, commentable par l'acteur. Quant à l'entretien d'explicitation, il vise en priorité la verbalisation de l'action, la mise en mots descriptive de l'action telle qu'elle a été effectivement mise en œuvre dans une tâche réelle. L'accès à une mémoire concrète, au vécu, aux procédures, à la conscience pré-réfléchie de l'action s'organise à partir de trace mnésique, affective et sensible. La singularité du moment n'est donc pas cristallisée par des enregistrements vidéo construits par le chercheur, mais elle est liée à la valeur émotionnelle de ce moment pour l'acteur.

D'autre part, trois types d'observation ont pu être identifiés à partir de l'analyse des résumés :

- L'observation en situation naturelle est une technique très répandue dans les recherches descriptives et compréhensives $(39,9 \%)$ : le chercheur se met à distance et évite que sa présence influence les comportements des acteurs étudiés, afin d'analyser des situations authentiques ;

- L'observation en situation négociée correspond davantage aux recherches à visée transformative ou sur l'efficacité de l'intervention (18\%): le chercheur souhaite 
observer un phénomène particulier et négocie avec le professionnel un dispositif (tâche d'apprentissage, organisation du cycle, modalités d'intervention...) pour évaluer les effets produits ;

- L'observation participante est plus rarement utilisée $(3,2 \%)$ : le chercheur s'intègre au groupe étudié et participe à la vie quotidienne avec ses membres, tout en conservant un recul suffisant pour être en mesure de l'observer et de l'étudier avec son regard extérieur.

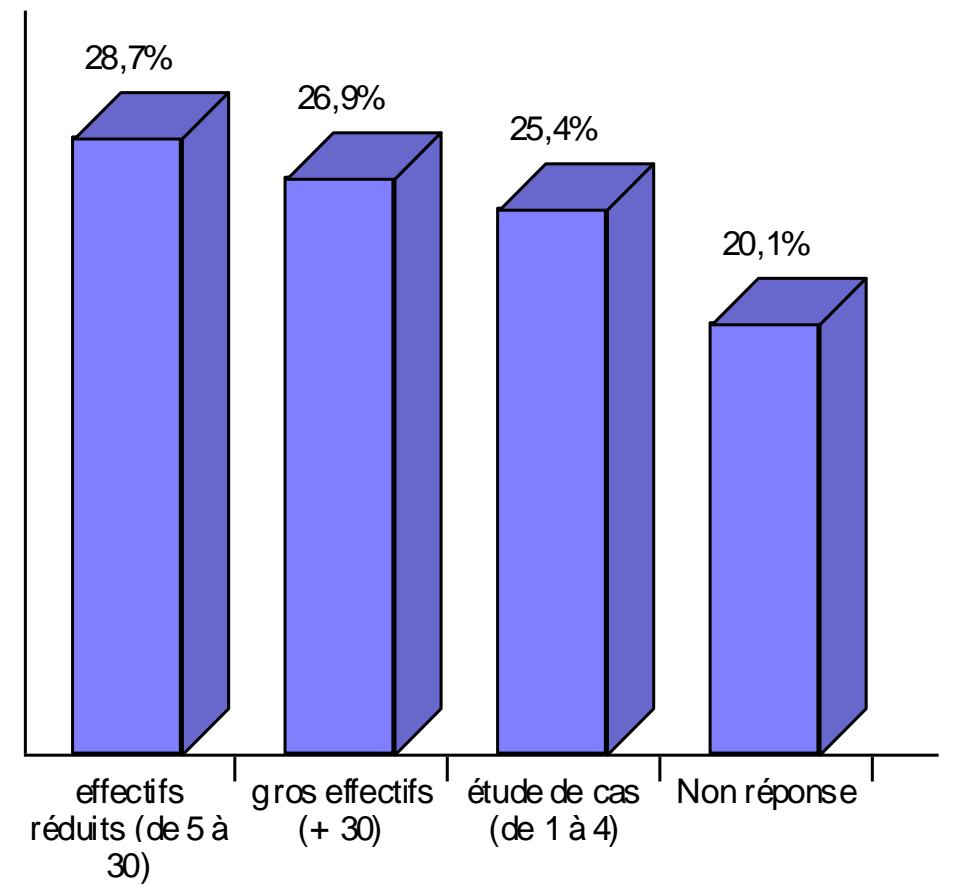

Figure 8. Méthodes de recueil de données utilisées dans les recherches

Enfin, la figure 8 confirme que les recherches francophones en intervention sont davantage qualitatives que quantitatives. En effet, plus d'une recherche sur deux s'intéresse à des groupes de moins de 30 individus, voire à des études de cas, en privilégiant l'observation et l'entretien. Cette tendance est inversée aux EU : même si les recherches qualitatives tendent à se développer récemment, l'utilisation des méthodes quantitatives dominent sans aucun doute les recherches en EPS (environ 18\% de recherches qualitatives selon Silverman \& Manson (2003) et Ward \& Ko (2006)). Nous constatons donc à nouveau des particularités dans les manières de concevoir les recherches en intervention dans le monde francophone et aux Etats-Unis. Notons enfin que de plus en plus de recherches sur l'intervention croisent méthodes quantitatives et méthodes qualitatives pour bénéficier de la complémentarité de ces deux types de méthodologies. 


\section{Conclusion}

Le but de cet article était de dresser un état des lieux des communications produites au cours des cinq premiers congrès ARIS de 2000 à 2008. Les résultats mettent en évidence des spécificités des recherches francophones sur l'intervention : elles sont essentiellement descriptives et compréhensives, utilisant les méthodes qualitatives. Cet observatoire des recherches sur l'intervention en sport montre aussi que les chercheurs mobilisent une diversité de champs scientifiques et de méthodologies pour analyser les pratiques d'intervention : ils reconstruisent et adaptent des outils conceptuels et méthodologiques en fonction des objets étudiés. Ainsi, les mêmes thématiques peuvent être étudiées à partir d'approches différentes, mais complémentaires. Nous voyons ici le signe d'une richesse indéniable, le croisement de regards différents permettant une meilleure compréhension des phénomènes éducatifs.

D'autre part, les professionnels semblent de moins en moins présents dans les congrès. Pourtant, la deuxième mission de l'association ARIS consiste à faciliter et développer les relations entre tous les chercheurs et les praticiens intéressés aux recherches dans le domaine des activités physiques et sportives, en suscitant des échanges et en soulignant la richesse des acquis professionnels. II apparaît donc aujourd'hui incontournable de favoriser les échanges entre professionnels et chercheurs. Les recherches restent peu connues des formateurs et des intervenants. La question de la diffusion des recherches auprès des professionnels semble de plus en plus problématique et mérite une attention toute particulière. Faire connaître ces résultats de recherches auprès des praticiens n'a rien d'évident, transformer les pratiques professionnelles encore moins. D'une part, les professionnels sont confrontés au quotidien à des problèmes divers, qu'ils doivent résoudre dans l'urgence. Une minorité d'entre eux peut se familiariser à la pratique de recherche et participer à des congrès. La recherche apparaît souvent comme éloignée du terrain, sans utilité pour la pratique. D'autre part, peu de chercheurs publient dans les revues professionnelles, non reconnues par le milieu universitaire et ont l'occasion de s'adresser spécialement aux professionnels pour présenter et discuter de leurs travaux. Pourtant, comment concevoir, dans le champ des pratiques d'intervention, la formation sans la recherche, ou la recherche sans la formation? Comment analyser des problèmes liés à l'intervention sans s'appuyer sur des théories? Et quel intérêt de construire des cadres théoriques si ce n'est pour questionner les pratiques? Tisser davantage de liens entre professionnels et chercheurs, réconcilier recherche et formation, dynamiser le travail collectif, réfléchir ensemble à des problèmes d'actualité liés aux pratiques sportives..., 
autant de missions de l'ARIS « urgentes et essentielles» (Durand, 2000), qui apparaissent incontournables dans le contexte actuel. II s'agit donc de repenser ce partenariat intervenants - formateurs - chercheurs en formation et en recherche, tout en analysant les sérieux problèmes épistémologiques et institutionnels posés par ce partenariat (Martinand, 2000). Le monde académique veut croire à un schéma de transfert - application du savoir produit par ou plutôt dans la pratique de recherche. Pourtant, les résultats de recherches ne sont pas directement applicables sur le terrain. En effet, les savoirs mobilisés par les enseignants et les entraîneurs ne sont pas une simple traduction linéaire de savoirs scientifiques qui seraient simplifiés, mais ils résultent de processus de transformation et de re-contruction difficiles à mettre en lumière. Leur appropriation passe par un métissage avec des croyances, des conceptions personnelles et une dialectique permanente avec l'expérience (Collinet, 2006). Pour Martinand (2000), «il faut donc plus qu'une recontextualisation, plus qu'une reformulation ou une traduction, il faut une reproblématisation dans le cadre d'une autre pratique ».

Enfin, ce premier inventaire des communications des congrès ARIS a permis de caractériser l'ensemble des communications présentées lors des congrès (730), mais il présente des limites, puisque nous nous sommes exclusivement appuyés sur les résumés des programmes scientifiques, pas suffisamment détaillés pour offrir un panorama complet des productions de l'ARIS. Cette enquête ouvre néanmoins plusieurs perspectives de travail intéressantes pour l'Observatoire des Recherches sur I'Intervention en Sport (ORIS), notamment des études qualitatives. D'ailleurs, I'ORIS a pour ambition de publier un ouvrage qui présentera une dizaine d'approches théoriques différentes pour analyser l'intervention (didactique, technologique, ergonomie cognitive, écologique, didactique clinique, socio-psychologique, sémiotique, socio-didactique, anthropologique).

Dans le second article de ce numéro, nous déclinerons les thématiques étudiées dans les principaux contextes analysés dans les recherches sur l'intervention, à savoir l'EPS, l'entraînement et la formation.

\section{Références}

Amade-Escot, C., \& Marsenach, J. (1993). Les orientations de la recherche en didactique de l'EPS. Revue française de pédagogie, 103, 33-42.

Amade-Escot, C., \& Amans-Passaga, C. (2006). Quality Physical Education : A review from Situated Research (1995-2005). Part One :"Curriculum and Content Issues". International Journal of Physical Education, a Review Publication, 43/4, 162-172. 
Artigue, M. (1990). Ingénierie didactique. Recherches en didactiques des mathématiques, 9(3), 283-307).

Astolfi, J.P. (1993). Trois paradigmes pour les recherches en didactique. Revue française de pédagogie, 103, 5-18.

Bardin, L. (2001). L'analyse de contenu. Paris : PUF.

Berger, P., \& Luckmann, T. (1986). La construction sociale de la réalité. Paris : Méridiens Klincksieck.

Berthelot, JM. (2008). L'emprise du vrai, connaissance scientifique et modernité. Paris : PUF.

Bouthier, D. (2001). L'intervention en EPS, panorama des axes et des types de recherches conduites ces dernières années. In J. Aubert, M. Durand \& R. Refuggi (Eds.), L'intervention dans le domaine des activités physiques et sportives: compétence(s) en mutation ? [CD-ROM]. Grenoble : IUFM.

Bouthier, D. (2005). "Sciences et techniques des APSA: quels rapports, vers quels métiers de l'intervention?». Conférence introductive au Forum International de l'EP et du Sport. Paris, 4-6 Novembre.

Bouthier, D., \& Durey, A. (1994). Technologie des APS, une voie possible en STAPS. Impulsions, 1, 94-125.

Bru, M. (2002). Pratiques enseignantes : des recherches à conforter et à développer. Revue française de pédagogie, 138, 63-71.

Collinet, C. (2006). Une analyse sociologique des savoirs scientifiques comme ressources possibles de l'action d'enseignants d'EPS et d'entraîneurs. Revue STAPS, 71, 115 $-133$.

David, B., Bouthier, D., Marsenach, J., \& Durey, A. (1999). French research into didactics and technology of physical activities and sports. An expanding new field. Instructional Sciences, 15, 148-162.

Durand, M., Euzet JP., \& Refuggi, R. (1998). L'intervention dans le domaine du sport et de la motricité humaine. Disponible sur internet au format PDF à l'adresse : http://www.unice.fr/ufrstaps/colloque_antibes/Durand/introantibes.htm (consulté le 5 Novembre 2008).

Durand, M. (2001). L'intervention dans le domaine du sport et de la motricité. In J. Aubert, M. Durand \& R. Refuggi (Eds.), L'intervention dans le domaine des activités physiques et sportives: compétence(s) en mutation? [CD-ROM]. Grenoble: IUFM.

Goodson, IF. (1988). The Making of Curriculum: Collected Essays. Lewes : Falmer. 
Hacking, I. (1999). The social construction of what ?. Cambridge : Harvad University Press.

Huberman, A \& Miles, B. (1991). Analyse de données qualitatives : recueil de nouvelles méthodes. Bruxelles : De Boeck Université.

Kirk, D., Macdonald, D., \& O'Sullivan, M. (2006). The Handbook of Physical Education. London: Sage.

Kulinna, P., Scrabis-Fletcher, K., Kodish, S., Phillips, S., \& Silverman, S. (2009). A decade of research literature in physical education pedagogy. JTPE, 28 (2), 119-140.

Lahire, B. (2005). L'esprit sociologique. Paris : La découverte.

Macdonald, D., Kirk, D., Metzler, M., Nilges, L. M., Schempp, P., \& Wright, J. (2002). It's all very well, in theory: Theoretical perspectives and their applications in contemporary pedagogical research. Quest, 54(2), 133-154.

Marsenach, J. \& Amade-Escot, C. (1993). Les orientations de la recherche en didactique de l'éducation physique et sportive. Revue française de pédagogie, 103, 33-42.

Martinand, J.L. (2000). Production, circulation et reproblématisation des savoirs. In M. Bru (Éd.), Les pratiques dans l'enseignement supérieur [CD-ROM]. Toulouse: université de Toulouse II.

Rix, G. (2006). La mise en perspective de modes d'investigation de l'activité humaine. In JM. Avenier \& C. Schmitt (Eds.), La construction de savoirs pour l'action (pp. 87 105). Paris : L'Harmattan.

Silverman, S., \& Skonie, R. (1997). Research on teaching in physical education : an analysis of published research. JTPE, 16 (3), 300-311.

Silverman, S., \& Manson, S. (2003). Research on teaching in physical education doctoral dissertations : a detailed investigation of focus, method, and analysis. JTPE, 22 (3), 280-297.

Theureau, J. (1992). Le cours d'action : analyse sémiologique. Essai d'une anthropologie cognitive située. Berne : Peter Lang.

Van Der Maren, J.M. (1995). Méthodes de recherche pour l'éducation. Paris, Bruxelles : De Boeck Université.

Vermersch, P. (1994). L'entretien d'explicitation. Paris : ESF.

Ward, P., \& Ko, B. (2006). Publication Trends in the Journal of Teaching in Physical Education from 1981 to 2005. JTPE, 25, 266-280.

Weil-Barais, A. (1997). Les méthodes en psychologie. Rosny : Bréal. 